\title{
The Free Boson Gas in a Rotating Bucket
}

\author{
J. T. Lewis \\ Dublin Institute for Advanced Studies, Dublin 4, Ireland
}

J. V. Pulè

Dipartiment tal-Matematika, Università Rjali ta' Malta, Malta

\section{§ 1. Introduction}

Putterman, Kac and Uhlenbeck [4] have proposed a purely quantum mechanical explanation for the origin of vortex lines in rotating He II, suggested by considering an ideal Boson gas in a rotating cylindrical bucket. Blatt and Butler [3] showed that a rotating ideal Boson gas undergoes phase transitions similar to those occurring in rotating He II. Their main result is that the total angular momentum $J$ of the gas, considered as a function of the angular velocity $\omega$ of the bucket, increases linearly between a sequence $\omega_{1}, \omega_{2}, \ldots$ of critical values of $\omega$. At a critical value of $\omega$ the angular momentum jumps by an amount $N_{0} \hbar$, where $N_{0}$ is the number of condensed particles. For $\omega$ between two critical values $\omega_{l}$ and $\omega_{l+1}$ we have

$$
J=\frac{1}{2}\left(N-N_{0}\right) m R^{2} \omega+N_{0} l \hbar,
$$

where $N$ is the total number of particles, $m$ is the mass of a particle and $R$ is the radius of the bucket. The contribution from the $N-N_{0}$ particles in the normal fluid is as if they were in solid body rotation. The contribution from the $N_{0}$ particles in the condensate is what one expects from a quantized vortex line of strength $i \hbar / m$ on the axis of the bucket.

The centrifugal density distortion in the normal fluid is negligible, since $\omega$ is assumed to be of order $\hbar / m R^{2}$. Then the kinetic energy of rotation when $m$ is the mass of a helium atom and $R$ is $1 \mathrm{~cm}$. is much smaller than $k T$ for temperatures around $1{ }^{\circ} \mathrm{K}$. This is not so for the condensate and these particles are pushed out to the rim of the contained for $\omega>\omega_{1}$. The reason for this behaviour can be seen informally as follows: The Bose condensation takes place into the single-particle state which is the ground state of the Hamiltonian $H=H_{0}-\omega J_{3}$ where $H_{0}$ is the free-particle Hamiltonian and $J_{3}$ is the operator corresponding to the component of angular momentum along the axis of the bucket. The eigenvalues of $H$ are $E_{k}-\hbar \omega l$ where $k=(n, l, m)$ stands for the three quantum numbers appropriate to the cylindrical geometry and the $E_{k}$ are the eigenvalues of $H_{0}$. We assume that the walls of the container are perfectly elastic so that the normal derivative of the wave-function is zero at the boundary. This is a reasonable assumption in thermal equilibrium (see [9]) but quite inappropriate for a discussion of the approach to equilibrium which would be necessary for an investigation of the formation of vortices (see [4]). The eigenfunctions of $H$ are the same as those 
of $H_{0}$ and are given by $K_{k} J_{|l|}\left(r_{l, n} r / R\right) e^{i l \theta} \cos (\pi m z / d)$ where $r_{l, n}$ is the $n$th nonnegative zero of $J_{|l|}^{\prime}$ and $d$ is the height of the container. For $\omega<\omega_{1}$ the ground state is $k=(1,0,0)$ and the condensate is uniformly distributed, since the corresponding eigenfunction is a constant. For $\omega=\omega_{1}$ there is an accidental degeneracy between the states $k=(1,0,0)$ and $k=(2,1,0)$ and for $\omega_{1}<\omega<\omega_{2}$ the ground state is $k=(2,1,0)$ with eigenfunction $\psi_{21}$ given by $k_{21} J_{1}\left(r_{21} r / R\right) e^{i \theta}$. The probability density $\left|\psi_{21}\right|^{2}$ has a maximum at the walls of the cylinder and the probability current density has a non-vanishing $\theta$-component $(\hbar / m r)\left|\psi_{21}\right|^{2}$ corresponding to the velocity distribution of a vortex line of strength $\hbar / m$ on the axis of rotation.

The purpose of this paper is to give rigorous justification for the above statements, proving the existence of condensation in the thermodynamic limit. The strategy is a modification of that given by $\mathrm{Kac}[1]$ in unpublished lectures and which has been worked out in detail for the free Boson gas in [2]. We assume the reader is familiar with this paper and where a proof is similar to one in [2] we omit it. Full details are to be found in [6].

The idea is to compute the generating functional of the representation of the cyclic representation of the canonical commutation relations corresponding to the state which is the thermodynamic limit of the canonical ensemble with fixed number density and fixed angular momentum density. The $n$-point functions can be read-off from the generating functional. However, the canonical ensemble is hard to handle directly and so the grand canonical ensemble for fixed mean number density and mean angular momentum density is used. The resulting generating functional is decomposed to get the canonical one at sharp values of the number density and angular momentum density.

In $\S 2$ we describe the procecure for passing to the thermodynamic limit and state as Theorem 1 our main result which describes the limiting form of the generating functional. As a step towards the proof of Theorem 1 we need to establish the existence of condensation; the essential result is stated as Theorem 2. The existence of condensation has already been studied by Blatt and Butler [3] and by Putterman, Kac and Uhlenbech [4] (see also [5]). In $\S 3$ we discuss our results before proving them in $\S 4$. The results about the representations of the CCR which we shall require are reviewed in [2] and we shall not repeat them here.

We are deeply indebted to Professor M. Kac for showing us his unpublished lecture notes on the subject of this paper. We are grateful to Dr. E. B. Davies for many stimulating discussions.

\section{§ 2. The Thermodynamic Limit of the Grand Canonical State}

Let $\Lambda^{1}$ be the cylindrical region in $\mathbb{R}^{3}$ of unit volume

$$
\Lambda^{1}=\left\{x \in \mathbb{R}^{3}: x_{1}^{2}+x_{2}^{2}<a^{2},\left|x_{3}\right|<\left(2 \pi a^{2}\right)^{-1}\right\}
$$

and for each $L>0$ let $\Lambda^{L}$ be the region

$$
\Lambda^{L}=\left\{x \in \mathbb{R}^{3}: L^{-1} x \in \Lambda^{1}\right\}
$$

and let $\mathfrak{H}_{L}$ be the space of square-integrable functions of $\Lambda^{L}$. Take $H_{L}$ to be the self-adjoint operator in $\mathfrak{H}_{L}$ determined by $-\frac{1}{2} \Delta$ in $\Lambda^{L}$ and the boundary condition 
$\partial \phi / \partial n=0$ on $\partial \Lambda^{L}$ where $\partial \phi / \partial n$ is the normal derivative. Let $J_{L}$ be the operator on $\mathfrak{H}_{L}$ corresponding to angular momentum about the $x_{3}$-axis. Let $N_{L}$ be the number operator on Fock space $\mathfrak{I}\left(\mathfrak{H}_{L}\right)$ and let $H_{L}^{F}$ and $J_{L}^{F}$ be the operators on $\mathfrak{J}\left(\mathfrak{H}_{L}\right)$ induced by $H_{L}$ and $J_{L}$ respectively.

Consider a free Boson gas in the bucket $\Lambda^{L}$. To describe the equilibrium state of such a system when rotating about the $x_{3}$-axis we take the density operator on $\mathfrak{I}\left(\mathfrak{H}_{L}\right)$ to be $\sigma_{\beta, z, \Omega}^{L}$ :

$$
\begin{aligned}
& \sigma_{\beta, z, \Omega}^{L}=\exp \left\{-\beta\left(H_{L}^{F}-\mu N_{L}^{F}-\Omega J_{L}^{F}\right)\right\} / Z_{\beta, z, \Omega}^{L}, \\
& Z_{\beta, z, \Omega}^{L}=\operatorname{trace}\left[\exp \left\{-\beta\left(H_{L}^{F}-\mu N_{L}^{F}-\Omega J_{L}^{F}\right)\right\},\right.
\end{aligned}
$$

where $\beta=1 / k T$ is the inverse temperature, $z=\exp \beta \mu$ is the fugacity and $\Omega$ can be interpreted as the angular velocity of the system. Let $W_{F}(h)$ be the free Weyl operators, then using standard results about Fock space we can show that the generating functional

$$
\mu_{\beta, z, \Omega}^{L}(h)=\left\langle W_{F}(h)\right\rangle=\operatorname{trace}\left[\sigma_{\beta, z, \Omega}^{L} W_{F}(h)\right]
$$

defined for $h \in \mathfrak{H}_{L}$ is given by

$$
\mu_{\beta, z, \Omega}^{L}(h)=\mu_{F}(h) \exp \left\{-\frac{1}{2} \mathscr{A}_{\beta, z, \Omega}^{L}(h, h)\right\}
$$

where

$$
\mathscr{A}_{\beta, z, \Omega}^{L}(h, h)=\left\langle h, z\left[\exp \left\{\beta\left(H_{L}-\Omega J_{L}\right)\right\}-z\right]^{-1} h\right\rangle .
$$

We wish to determine the limit as $L \rightarrow \infty$ of $\mathscr{A}_{\beta, z, \Omega}^{L}(h, h)$ when the mean density $\bar{\varrho}$ and the mean density $\bar{\lambda}$ of angular momentum in the $x_{3}$-direction are held fixed.

Let $\left\{\phi_{k}^{L}: k=1,2,3, \ldots\right\}$ be a complete orthonormal set in $\mathfrak{H}_{L}$ such that

$$
\begin{aligned}
H_{L} \phi_{k}^{L} & =E_{k}^{L} \phi_{k}^{L}, \\
J_{L} \phi_{k}^{L} & =l_{k} \phi_{k}^{L},
\end{aligned}
$$

where the ordering is taken so that

$$
E_{1}^{L}-\Omega l_{1} \leqq E_{2}^{L}-\Omega l_{2} \leqq \ldots .
$$

Then $\phi_{k}^{L}$ is given in terms of $\phi_{k}=\phi_{k}^{1}$ by

$$
\begin{aligned}
\phi_{k}^{L}(x) & =L^{-3 / 2} \phi_{k}\left(L^{-1} x\right), \\
E_{k}^{L} & =L^{-2} E_{k} .
\end{aligned}
$$

For the set $\left\{\phi_{k}\right\}$ we shall take:

$$
K_{l, n} J_{|l|}\left(r_{l, n} a^{-1} r\right) e^{i l \theta} \cos \left(\pi^{2} a^{2} m x_{3}+\pi m / 2\right),
$$

where $l=0, \pm 1, \pm 2, \ldots, m=0,1,2, \ldots, n=1,2, \ldots, r_{l, n}$ is the $n$th non-negative zero of $J_{|l|}^{\prime}$ in increasing order and $K_{0,0}=1$ and for $|l|+n \geqq 1$

$$
K_{l, n}=2^{1 / 2} r_{l, n}\left[2 \pi a^{2}\left(r_{l, n}^{2}-l^{2}\right)^{1 / 2} J_{|l|}\left(r_{l, n}\right)\right]^{-1} .
$$

Consequently the set of energies $\left\{E_{k}\right\}$ is given by

$$
\left(2 a^{2}\right)^{-1} r_{l, n}^{2}+2^{-1} \pi^{4} a^{4} m^{2} \text {. }
$$


We shall also require the sequence of positive real numbers $\left\{\omega_{k}\right\}$ given by $\omega_{0}=0$ and for $l \geqq 1$

$$
\omega_{l}=\left(2 a^{2}\right)^{-1}\left(r_{l, 1}^{2}-r_{l-1,1}^{2}\right) \text {. }
$$

The number operator $N_{L}$ is given by

$$
N_{L}=\sum_{k=1}^{\infty} \Psi^{*}\left(\phi_{k}^{L}\right) \Psi\left(\phi_{k}^{L}\right)
$$

and the number of particles in the $k$ th level is given by the operator

$$
n_{k}^{L}=\Psi^{*}\left(\phi_{k}^{L}\right) \Psi\left(\phi_{k}^{L}\right) .
$$

Using (2.1) we have

$$
\begin{aligned}
\left\langle n_{k}^{L}\right\rangle & =\operatorname{trace}\left(\sigma_{\beta, z, \Omega}^{L} n_{k}^{L}\right) \\
& =\mathscr{A}_{\beta, z, \Omega}^{L}\left(\phi_{k}^{L}, \phi_{k}^{L}\right) \\
& =z /\left(\exp \left\{\beta\left(E_{k}^{L}-\Omega l_{k}\right)\right\}-z\right)
\end{aligned}
$$

and

$$
\left\langle N_{L}\right\rangle=\sum_{k=1}^{\infty} z\left[\exp \left\{\beta\left(E_{k}^{L}-\Omega l_{k}\right)\right\}-z\right]^{-1} .
$$

Similarly

$$
\left\langle J_{L}\right\rangle=\sum_{k=1}^{\infty} z l_{k}\left[\exp \left\{\beta\left(E_{k}^{L}-\Omega l_{k}\right)\right\}-z\right]^{-1} .
$$

We require $\left\langle N_{L}\right\rangle$ to have the value $\varrho L^{3}$ where $\varrho$ is the mean density and $\left\langle J_{L}\right\rangle$ to have the value $\bar{\lambda} L^{3}$ where $\bar{\lambda}$ is the mean angular momentum density. This determines $z$ and $\Omega$ as functions of $L$ through the constraints

$$
\bar{\varrho}=L^{-3} \sum_{k=1}^{\infty} z(L)\left[\exp \left\{\beta\left(E_{k}^{L}-\Omega(L) l_{k}\right\}-z(L)\right]^{-1}\right.
$$

and

$$
\bar{\lambda}=L^{-3} \sum_{k=1}^{\infty} z(L) l_{k}\left[\exp \left\{\beta\left(E_{k}^{L}-\Omega(L) l_{k}\right\}-z(L)\right]^{-1} .\right.
$$

Our objective is to find

$$
\mu_{\beta, \bar{\varrho}, \bar{\lambda}}(h)=\lim _{L \rightarrow \infty} \mu_{\beta, z(L), \Omega(L)}^{L}(h)
$$

for $h$ in some dense subset of $L^{2}(\mathbb{R})$. It is sufficient to determine

$$
\mathscr{A}_{\beta, \bar{Q}, \bar{\lambda}}(h, h)=\lim _{L \rightarrow \infty} \mathscr{A}_{\beta, z(L), \Omega(L)}^{L}(h, h) .
$$

Then

$$
\mu_{\beta, \bar{\varrho}, \bar{\lambda}}(h)=\mu_{F}(h) \exp \left\{-\frac{1}{2} \mathscr{A}_{\beta, \bar{\varrho}, \bar{\lambda}}(h, h)\right\} .
$$

We interpret $\mu_{\beta, \bar{\varrho}, \bar{\lambda}}(h)$ as the generating functional for a representation of the CCR for an infinite free Boson gas with mean density $\bar{\varrho}$ and mean angular momentum density $\bar{\lambda}$. Let $g_{\alpha}$ be defined on the interval $[0,1]$ by

$$
g_{\alpha}(z)=\sum_{n=1}^{\infty} n^{-\alpha} z^{n}, \quad \alpha>1
$$


$z \mapsto(2 \pi \beta)^{-3 / 2} g_{3 / 2}(z)$ is continuous on $[0,1]$ and increases monotonically to a maximum $\varrho_{c}$ at $z=1$ so that for $\varrho \leqq \varrho_{c}$, the equation

$$
\bar{\varrho}=(2 \pi \beta)^{-3 / 2} g_{3 / 2}(\theta)
$$

has a unique root $\theta(\bar{\varrho})$.

Let $\mathscr{D}$ be the space of $C^{\infty}$ functions on $\mathbb{R}^{3}$ having compact support.

Theorem 1. The quadratic form $\mathscr{A}_{\beta, \bar{\varrho}, \bar{\lambda}}$ is given on $\mathscr{D}$ by

$$
\begin{aligned}
& \mathscr{A}_{\beta, \bar{\varrho}, \bar{\lambda}}(h, h)=\left\{\begin{array}{l}
\left\langle h, f_{\theta(\varrho)} h\right\rangle, \quad \varrho \leqq \varrho_{c}, \\
\left(\bar{\varrho}-\varrho_{c}\right) G\left(\left(\bar{\lambda}-\omega_{1} a^{2} \varrho_{c} / 2\right) /\left(\bar{\varrho}-\varrho_{c}\right)\right)|\hat{h}(0)|^{2}
\end{array}\right. \\
& \left.+\left\langle h, f_{1} h\right\rangle, \varrho\right\rangle \varrho_{c},
\end{aligned}
$$

where

$$
\left(f_{z} h\right)(s)=\int_{\mathbb{R}^{3}} f_{z}\left(\|x-y\|_{1}\right) h(y) d y,
$$

with

$$
f_{z}(s)=(2 \pi \beta)^{-3 / 2} \sum_{n=1}^{\infty} e^{-s^{2} / 2 n \beta} n^{-3 / 2} z^{n},
$$

and

$$
G(t)=\left\{\begin{array}{l}
1, t<0 \\
1-t ; 0 \leqq t \leqq 1 \\
0, t>1
\end{array}\right.
$$

A necessary preliminary to the proof of this theorem is the determination of the asymptotic behaviour of the solutions $z(L)$ and $\Omega(L)$ of the constraint Eqs. (2.6), (2.7) for large values of $L$, or of the solutions $\zeta(L)$ and $\omega(L)$ of the transformed equations

$$
\begin{aligned}
& \bar{\varrho}=L^{-3} \sum_{k=1}^{\infty} \zeta(L)\left(\exp \left\{\beta L^{-2} \eta_{k}(\omega(L))\right\}-\zeta(L)\right)^{-1}, \\
& \bar{\lambda}=L^{-3} \sum_{k=1}^{\infty} l_{k}(\omega(L)) \zeta(L)\left(\exp \left\{\beta L^{-2} \eta_{k}(\omega(L))\right\}-\zeta(L)\right)^{-1},
\end{aligned}
$$

where

$$
\begin{aligned}
\omega(L) & =L^{-2} \Omega(L), \\
\varepsilon_{k}(\omega) & =E_{k}-\omega l_{k}, \\
\zeta(L) & =z(L) \exp \left\{-\beta L^{-2} \varepsilon_{1}(\omega(L))\right\}, \\
\eta_{k}(\omega) & =\varepsilon_{k}(\omega)-\varepsilon_{1}(\omega),
\end{aligned}
$$

so that $0 \leqq \eta_{1}(\omega) \leqq \eta_{2}(\omega) \leqq \ldots$. Some of the properties of the set $\left\{\varepsilon_{k}\right\}$ we shall require are given without proof in Lemma 4 in the appendix. We write $l_{k}(\omega(L))$ because the ordering depends on $\omega$. One can show [6] that $\omega_{l}<\omega_{l+1}$ for all $l \geqq 0$ and for $\omega \in\left[\omega_{l}, \omega_{l+1}\right) \varepsilon_{1}(\omega)=\left(2 a^{2}\right)^{-1} r_{l, 1}^{2}-\omega l$ so that $l_{1}(\omega)=l$. Thus $l_{1}(\omega)$ is an increasing step function with steps of unit height starting at zero. Also from Lemma $4 l_{2}\left(\omega_{l}\right)=$ $l-1$ for $l \geqq 1$ and $l_{2}\left(\omega_{0}\right)=0$ so that

$$
\varepsilon_{1}\left(\omega_{l}\right)=\varepsilon_{2}\left(\omega_{l}\right)
$$


and therefore $\eta_{1}\left(\omega_{l}\right)=\eta_{2}\left(\omega_{l}\right)=0$. Thus the first two terms of (2.11) and (2.12) become infinitely large as $\zeta$ approaches 1 , if $\omega$ approaches one of the values $\omega_{l}$. This means that we have to isolate the first two terms rather than just the first as we did in [2]. Therefore let

$$
\begin{aligned}
& a_{L}(\omega, \zeta)=L^{-3} \zeta(1-\zeta)^{-1}+L^{-3} \zeta\left(\exp \left\{\beta L^{-2} \eta_{2}(\omega)\right\}-\zeta\right)^{-1}, \\
& c_{L}(\omega, \zeta)=L^{-3} \zeta\left(\exp \left\{\beta L^{-2} \eta_{2}(\omega)\right\}-\zeta\right)^{-1}
\end{aligned}
$$

and

$$
b_{L}(\omega, \zeta)=L^{-3} \zeta l_{1}(\omega)(1-\zeta)^{-1}+L^{-3} \zeta l_{2}(\omega)\left(\exp \left\{\beta L^{-2} \eta_{2}(\omega)\right\}-\zeta\right)^{-1} .
$$

The properties of $\zeta(L)$ and $\omega(L)$ are given by the following theorem. Since $\left\langle n_{k}^{L}\right\rangle$ must be positive and finite for all $k$ we require $0 \leqq \zeta(L) \leqq 1$.

Theorem 2. For $\bar{\varrho} \leqq \varrho_{c}, a_{L}(\omega(L), \zeta(L)) \rightarrow 0, \omega(L) \rightarrow 2 \bar{\lambda} / a^{2} \bar{\varrho}$ and $\zeta(L) \rightarrow \theta(\bar{\varrho})$ where $\theta(\varrho)$ is the root of the equation

$$
\bar{\varrho}=(2 \pi \beta)^{-3 / 2} g_{3 / 2}(\theta) \text {. }
$$

For $\bar{\varrho}>\varrho_{c}, a_{L}(\omega(L), \zeta(L)) \rightarrow \bar{\varrho}-\varrho_{c}, \zeta(L) \rightarrow 1$ and

(a) if the line $y=\left(\bar{\lambda}-\omega a^{2} \varrho_{c} / 2\right) /\left(\bar{\varrho}-\varrho_{c}\right)$ intersects the step function $y=l_{1}(\omega)$ at $\omega^{\prime} \in\left(\omega_{l}, \omega_{l+1}\right)$ for some $l \geqq 1$ then $\omega(L) \rightarrow \omega^{\prime}$ and $c_{L}(\omega(L), \zeta(L)) \rightarrow 0$, while

(b) if the line passes through the gap at $\omega=\omega_{l}$ a distance $t$ from the bottom of the gap then $\omega(L) \rightarrow \omega_{l}$ and

$$
c_{l}(\omega(L), \zeta(L)) \rightarrow[t \wedge(1-t)]\left(\bar{\varrho}-\varrho_{c}\right) .
$$

Also in case (a) $l_{1}(\omega(L)) \rightarrow l$ and in case (b),

$$
\begin{array}{lll}
l_{1}(\omega(L)) \rightarrow l, l_{2}(\omega(L)) \rightarrow l-1 & \text { if } & \frac{1}{2}<t \leqq 1, \\
l_{1}(\omega(L)) \rightarrow l-1, l_{2}(\omega(L)) \rightarrow l & \text { if } & 0 \leqq t<\frac{1}{2} .
\end{array}
$$

\section{§ 3. Discussion of Results}

To make the results of Theorem 2 clearer we have given a graphical representation (Fig. 1); they are in fact very simple.

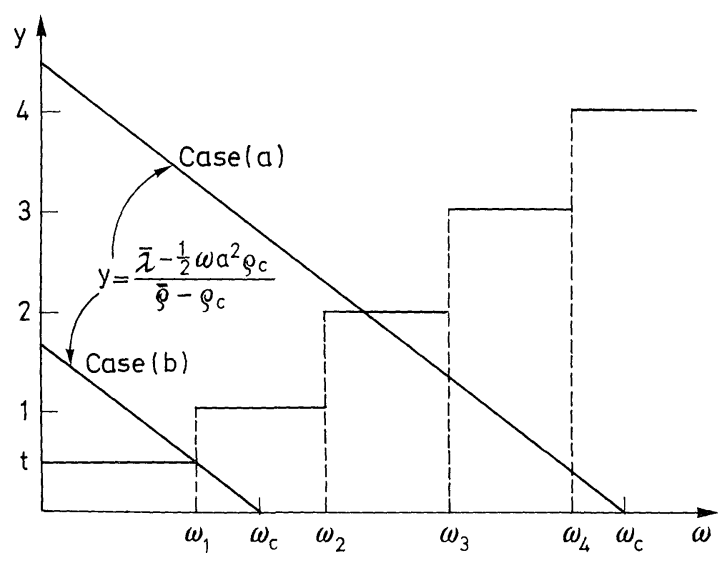


We must remember that

$$
\begin{aligned}
& L^{-3}\left(\left\langle n_{1}^{L}\right\rangle+\left\langle n_{2}^{L}\right\rangle\right)=a_{L}(\omega(L), \zeta(L)), \\
& L^{-3}\left\langle n_{1}^{L}\right\rangle=a_{L}(\omega(L), \zeta(L))-c_{L}(\omega(L), \zeta(L)),
\end{aligned}
$$

and

$$
L^{-3}\left\langle n_{2}^{L}\right\rangle=c_{L}(\omega(L), \zeta(L)) .
$$

For $\bar{\varrho} \leqq \varrho_{c}$ all three quantities tend to zero as $L \rightarrow \infty$. Therefore, there is no condensation. For $\bar{\varrho}>\varrho_{c}$ if $\bar{\lambda}, \bar{\varrho}$ are as in case (a) then

$$
L^{-3}\left\langle n_{1}^{L}\right\rangle \rightarrow\left(\bar{\varrho}-\varrho_{c}\right)
$$

and

$$
L^{-3}\left\langle n_{2}^{L}\right\rangle \rightarrow 0 .
$$

That is, there is condensation in one state corresponding to the quantum numbers $(l, 1,0)$. Suppose on the other hand we have case (b) with $\frac{1}{2}<t<1$ for example. Then both $L^{-3}\left\langle n_{1}^{L}\right\rangle$ and $L^{-3}\left\langle n_{2}^{L}\right\rangle$ have a non-vanishing limit; that is, there is condensation in both states $(l, 1,0),(l-1,1,0)$ and the ratio of the amount of gas in each state is given by

$$
\lim _{L \rightarrow \infty}\left\langle n_{1}^{L}\right\rangle /\left\langle n_{2}^{L}\right\rangle=(1-t) / t
$$

If we look at case (a) again and let

$$
\omega_{\infty}=\lim _{L \rightarrow \infty} \omega(L)
$$

we see that $\bar{\lambda}, \bar{\varrho}$ and $\omega_{\infty}$ are related by

$$
\bar{\lambda}=l_{1}\left(\omega_{\infty}\right)\left(\bar{\varrho}-\varrho_{c}\right)+\frac{1}{2} \omega_{\infty} a^{2} \varrho_{c} .
$$

This is very reasonable since the first term on the right hand side represents the angular momentum density of a system of particles with density $\left(\bar{\varrho}-\varrho_{c}\right)$ each particle having angular momentum $l_{1}\left(\omega_{\infty}\right)$ and the second term can be written as the limit, as $L \rightarrow \infty$, of $\frac{1}{2} \omega_{\infty} L^{-2}\left(a L^{2}\right) \varrho_{c}$ which is the angular momentum density of a classical system in $\Lambda^{L}$ with density $\varrho_{c}$ and rotating with velocity $\omega_{\infty} L^{-2}$. Because $r_{l, 1}=l+b l^{1 / 3}+O\left(l^{-1 / 3}\right)$ where $b>0,[7], \omega_{l} l^{-2} \rightarrow a^{-2}$ as $l \rightarrow \infty$; this means that the relation (3.1) for large $l$ becomes approximately

$$
\bar{\lambda}=\omega_{\infty} a^{2}\left(\bar{\varrho}-\varrho_{c}\right)+\frac{1}{2} \omega_{\infty} a^{2} \varrho_{c} .
$$

There the moment of inertia of the gas is larger than the classical. These results agree with Blatt and Butler's [3] who obtained them using approximate methods.

We now consider the generating functional. From Theorem 1 we see that if $\bar{\varrho}>\varrho_{c}$ for some values of $\bar{\lambda}$ there is no off-diagonal long range order in the thermodynamic limit although there is Bose-Einstein condensation. We explain this as follows. For simplicity we take $\omega_{\infty} \neq \omega_{l}$. Let $\left\langle\Psi^{*}(x) \Psi(y)\right\rangle$ denote the kernel of the quadratic form $\left\langle\Psi^{*}\left(h_{1}\right) \Psi\left(h_{2}\right)\right\rangle$ so that

$$
\left\langle\Psi^{*}\left(h_{1}\right) \Psi\left(h_{2}\right)\right\rangle=\int_{\mathbb{R}^{3} \times \mathbb{R}^{3}} h_{1}(x)\left\langle\Psi^{*}(x) \Psi(y)\right\rangle h_{2}(y) d x d y .
$$


Then we can interpret $\varrho(x)=\left\langle\Psi^{*}(x) \Psi(x)\right\rangle$ as the density at $x$. For $\left(\bar{\lambda}-\frac{1}{2} \omega_{1} a^{2} \varrho_{c}\right) /$ $\left(\bar{\varrho}-\varrho_{c}\right) \leqq 1$ we have $\varrho(x)=\left(\bar{\varrho}-\varrho_{c}\right)+\varrho_{c}=\bar{\varrho}$, while for $\left(\bar{\lambda}-\frac{1}{2} \omega_{1} a^{2} \varrho_{c}\right) /\left(\bar{\varrho}-\varrho_{c}\right)>1$, $\varrho(x)=\varrho_{c}$. Thus $\varrho(x)<\bar{\varrho}$ in the second case. This is because the condensate moves to the boundary. We can see this in another way. Let us show the $\omega$ dependence of the ordering of $\left\{\phi_{k}^{L}\right\}$ by writing $\left\{\phi_{k, \omega}^{L}\right\}$ and let us look at that part of $\mathscr{A}^{L}$ which corresponds to the condensate

$$
\mathscr{A}_{c}^{L}(h, h)=\zeta(L)(1-\zeta(L))^{-1}\left|\left\langle h, \phi_{1, \omega(L)}^{L} h\right\rangle\right|^{2}
$$

which gives

$$
\begin{aligned}
\varrho_{c}^{L}(x) & =\left\langle\Psi^{*}(x) \Psi(x)\right\rangle_{c}^{L} \\
& =\zeta(L)(1-\zeta(L))^{-1}\left|\phi_{1, \omega(L)}^{L}(x)\right|^{2} \\
& =L^{-3} \zeta(L)(1-\zeta(L))^{-1}\left|\phi_{1, \omega(L)}(x / L)\right|^{2}
\end{aligned}
$$

so that for $\left(\bar{\lambda}-\frac{1}{2} \omega_{1} a^{2} \varrho_{c}\right) /\left(\bar{\varrho}-\varrho_{c}\right)>1, \varrho_{c}^{L}(x) \rightarrow 0$ as $L \rightarrow \infty$. However for $0 \leqq \kappa \leqq 1$ consider the density of the condensate at $x^{L}=\left(a \kappa L, \theta, x_{3}\right)$ in cylindrical polar co-ordinates. Then

$$
\begin{aligned}
\varrho_{c}^{L}\left(x^{L}\right)= & L^{-3} \zeta(L)(1-\zeta(L))^{-1} K_{l_{1}(\omega(L)), 1} J_{l_{1}(\omega(L))}^{2}\left(\kappa r_{l_{1}(\omega(L)), 1}\right) \\
& \rightarrow\left(\bar{\varrho}-\varrho_{c}\right) K_{l_{1}\left(\omega_{\infty}\right), 1} J_{l_{1}\left(\omega_{\infty}\right), 1}^{2}\left(\kappa r_{l_{1}\left(\omega_{\infty}\right), 1}\right)
\end{aligned}
$$

which is equal to $\left(\bar{\varrho}-\varrho_{c}\right)$ for $l_{1}\left(\omega_{\infty}\right)=0$ but for $l_{1}\left(\omega_{\infty}\right)>0$ it increases monotonically from zero at $\kappa=0$ to a maximum at $\kappa=1$.

If we define the condensate current density $j_{c}^{L}(x)$ by

$$
j_{c}^{L}(x)=-i / 2\left\langle\Psi^{*}(x) \operatorname{grad} \Psi(x)-\operatorname{grad} \Psi^{*}(x) \Psi(x)\right\rangle_{c}^{L}
$$

where $(\operatorname{grad} \Psi)(h)=-\Psi(\operatorname{grad} h)$ and $\left(\operatorname{grad} \Psi^{*}\right)(h)=-\Psi^{*}(\operatorname{grad} h)$ then it is easy to check that

$$
j_{c}^{L}(x)=\varrho_{c}^{L}(x) r^{-1} l_{1}(\omega(L)) \hat{n}_{\theta}
$$

where $\hat{n}_{\theta}$ is the unit vector to the plane $\theta=$ constant. Therefore

$$
v_{c}^{L}(x)=j_{c}^{L}(x) / \varrho_{c}^{L}(x)=r^{-1} l_{1}(\omega(L)) \hat{n}_{\theta}
$$

and

$$
v_{c}(x)=\lim _{L \rightarrow \infty} v_{c}^{L}(x)=r^{-1} l_{1}\left(\omega_{\infty}\right) \hat{n}_{\theta}
$$

which is the velocity distribution of a vortex of strength $l_{1}\left(\omega_{\infty}\right)$. In c.g.s. units the strength of the vortex is $h l_{1}\left(\omega_{\infty}\right) / m$ where $m$ is the particle mass and $h$ is Planck's constant, which agrees with the Onsager-Feynman hypothesis about the quantization of the circulation in a superfluid [8].

\section{§4. Proof of the Theorems}

Let $\zeta_{1}(L, \omega)$ and $\zeta_{2}(L, \omega)$ be the solutions of

$$
\bar{\varrho}=L^{-3} \sum_{k=1}^{\infty} \zeta_{1}(L, \omega)\left(\exp \left\{\beta L^{-2} \eta_{k}(\omega)\right\}-\zeta_{1}(L, \omega)\right)^{-1}
$$

and

$$
\bar{\lambda}=L^{-3} \sum_{k=1}^{\infty} l_{k}(\omega) \zeta_{2}(L, \omega)\left(\exp \left\{\beta L^{-2} \eta_{k}(\omega)\right\}-\zeta_{2}(L, \omega)\right)^{-1}
$$


respectively, each equation being taken separately. Then $\omega(L)$ is given by the solution of the equation

$$
\zeta_{1}(L, \omega(L))=\zeta_{2}(L, \omega(L))
$$

and $\zeta(L)$ by

$$
\zeta(L)=\zeta_{1}(L, \omega(L))=\zeta_{2}(L, \omega(L)) .
$$

To obtain the asymptotic properties of $\zeta_{1}(L, \omega)$ and $\zeta_{2}(L, \omega)$ we use a modified form of Kac's method used in [2].

The Green's function for the equation

$$
\partial \psi / \partial t+\frac{1}{2} \Delta \psi-i \omega \partial \psi / \partial \theta=0
$$

in $\Lambda^{1}$ subject to the boundary condition $\partial \psi / \partial n=0$ is given in cylindrical polar co-ordinates by

$$
\begin{aligned}
& G_{\omega}\left(r, \theta, x_{3} ; r^{\prime}, \theta^{\prime}, x_{3}^{\prime} ; t\right) \\
& =\sum_{k=1}^{\infty} e^{-t \varepsilon_{k}(\omega)} \overline{\phi_{k, \omega}\left(r, \theta, x_{3}\right)} \phi_{k, \omega}\left(r^{\prime}, \theta^{\prime}, x_{3}^{\prime}\right) .
\end{aligned}
$$

For small $t$, the effect of the boundary is not felt and we expect

$$
\begin{aligned}
& G_{\omega}\left(r, \theta, x_{3} ; r^{\prime}, \theta^{\prime}, x_{3}^{\prime} ; t\right) \sim G_{\omega}^{0}\left(r, \theta, x_{3} ; r^{\prime}, \theta^{\prime}, x_{3}^{\prime} ; t\right) \\
& =(2 \pi t)^{-3 / 2} \exp \left\{-(2 t)^{-1}\left[\left(x_{3}-x_{3}^{\prime}\right)^{2}+r^{2}+r^{\prime 2}-2 r r^{\prime} \cos \left(\theta-\theta^{\prime}+i \omega t\right)\right]\right\} .
\end{aligned}
$$

Putting $\left(r, \theta, x_{3}\right)=\left(r^{\prime}, \theta^{\prime}, x_{3}^{\prime}\right)$ and integrating over $\Lambda^{1}$ we get

$$
\begin{aligned}
\sum_{k=1}^{\infty} e^{-t \varepsilon_{k}(\omega)} & \sim(2 \pi t)^{-3 / 2} 2 a^{-2} \int_{0}^{a} \exp \left\{(2 / t) r^{2} \sinh ^{2}(\omega t / 2)\right\} r d r \\
& \sim(2 \pi t)^{-3 / 2} .
\end{aligned}
$$

Now by interchanging the order of summation we obtain

$$
L^{-3} \sum_{k=1}^{\infty} \zeta\left(\exp \left\{\beta L^{-2} \eta_{k}(\omega)\right\}-\zeta\right)^{-1}=\sum_{n=1}^{\infty} \zeta^{n} S_{L, \omega}(n \beta)
$$

where

$$
\begin{aligned}
S_{L, \omega}(s)= & L^{-3} \sum_{k=1}^{\infty} e^{-s L^{-2} \eta_{k}(\omega)} \\
= & L^{-3} e^{s L^{-2} \varepsilon_{1}(\omega)} \sum_{k=1}^{\infty} e^{-s L^{-2} \varepsilon_{k}(\omega)} \\
& \sim(2 \pi s)^{-3 / 2}
\end{aligned}
$$

for large $L$ so that

$$
L^{-3} \sum_{k=1}^{\infty} \zeta\left(\exp \left\{\beta L^{-2} \eta_{k}(\omega)\right\}-\zeta\right)^{-1} \sim(2 \pi \beta)^{-3 / 2} g_{3 / 2}(\zeta)
$$

and therefore $\zeta_{1}(L, \omega) \sim \theta(\bar{\varrho})$. For $\bar{\varrho} \geqq \varrho_{c}$ we have to go back to the series (4.1). The first two terms dominate as $\zeta$ gets close to 1 and so we remove them and define

$$
S_{L, \omega}^{\prime}(s)=L^{-3} \sum_{k=3}^{\infty} e^{-s L^{-2} \eta_{k}(\omega)}
$$

to prove

Lemma 1. Let $f_{L, \omega}(\zeta)=\sum_{n=1}^{\infty} \zeta^{n} S_{L, \omega}^{\prime}(n \beta)$. Then for each $N, f_{L, \omega}(\zeta) \rightarrow$ $(2 \pi \beta)^{-3 / 2} g_{3 / 2}(\zeta)$ uniformly for $\zeta \in[0,1], \omega \in\left[0, \omega_{N}\right]$ as $L \rightarrow \infty$.

Using this we are able to prove the following theorem. 
Theorem 3. For $\bar{\varrho}<\varrho_{c}, a_{L}\left(\omega, \zeta_{1}(L, \omega)\right) \rightarrow 0$ and $\zeta_{1}(L, \omega) \rightarrow \theta(\bar{\varrho})$ uniformly in $\left[0, \omega_{N}\right]$ and for $\bar{\varrho} \geqq \varrho_{c}, a_{L}\left(\omega, \zeta_{1}(L, \omega)\right) \rightarrow \bar{\varrho}-\varrho_{c}$ and $\zeta_{1}(L, \omega) \rightarrow 1$ uniformly in $\left[0, \omega_{N}\right]$.

Furthermore if $\omega_{l}<\omega^{\prime}<\omega^{\prime \prime}<\omega_{l+1}$ then $c_{L}\left(\omega, \zeta_{1}(L, \omega)\right) \rightarrow 0$ uniformly on $\left[\omega^{\prime}, \omega^{\prime \prime}\right]$.

Lemma 1 and Theorem 3 are very similar to Lemma 1 and Theorem 1 in [2] and therefore we shall not prove them. The following estimate is used in the proof of Lemma 1. Let $g_{\omega}\left(x, x^{\prime} ; t\right)=G_{\omega}\left(x, x^{\prime} ; t\right)-G_{\omega}^{0}\left(x, x^{\prime} ; t\right)$; then for each $T<\infty$ and $\Omega<\infty$ there is a constant $C(T, \Omega)$ such that for $t<T, \omega \leqq \Omega$,

$$
\left|g_{\omega}\left(x, x^{\prime} ; t\right)\right| \leqq t^{-5 / 4} C(T, \Omega)\left[a-\left(x_{1}^{2}+x_{2}^{2}\right)^{1 / 2}\right]^{-3 / 4} l\left(x_{3}\right)^{-3 / 4}
$$

where $l\left(x_{3}\right)=\left[\left(2 \pi a^{2}\right)^{-1}-x_{3}\right] \wedge\left[\left(2 \pi a^{2}\right)^{-1}+x_{3}\right][6]$.

The last statement of Theorem 3 is proved as follows. One can show (Lemma 4) that there is a $\delta>0$ such that if $\omega \in\left[\omega^{\prime}, \omega^{\prime \prime}\right], \eta_{2}(\omega)>\delta$. Thus

$$
c_{L}\left(\omega, \zeta_{1}(L, \omega)\right)<L^{-3}\left[\exp \left\{\beta L^{-2} \delta\right\}-1\right]^{-1} \rightarrow 0
$$

uniformly in $\left[\omega^{\prime}, \omega^{\prime \prime}\right]$ as $L \rightarrow \infty$.

To obtain the asymptotic form of the sum

$$
L^{-3} \sum_{k=1}^{\infty} l_{k}(\omega) \zeta\left(\exp \left\{\beta L^{-2} \eta_{k}(\omega)\right\}-\zeta\right)^{-1}
$$

we note that

$$
\begin{aligned}
& i \partial G_{\omega} / \partial \theta\left(r, \theta, x_{3} ; r^{\prime}, \theta^{\prime}, x_{3}^{\prime} ; t\right) \\
& =\sum_{k=1}^{\infty} l_{k}(\omega) e^{-t \varepsilon_{k}(\omega)} \overline{\phi_{k, \omega}\left(r, \theta, x_{3}\right)} \phi_{k, \omega}\left(r^{\prime}, \theta^{\prime}, x_{3}^{\prime}\right) .
\end{aligned}
$$

Then using the same method as before we see that

$$
\sum_{k=1}^{\infty} l_{k}(\omega) e^{-t \varepsilon_{k}(\omega)} \sim \frac{1}{2} \omega a^{2}(2 \pi t)^{-3 / 2}
$$

for small $t$ so that for large $L$

$$
L^{-3} \sum_{k=1}^{\infty} l_{k}(\omega) \zeta\left(\exp \left\{\beta L^{-2} \eta_{k}(\omega)\right\}-\zeta\right)^{-1} \sim \frac{1}{2} \omega a^{2}(2 \pi \beta)^{-3 / 2} g_{3 / 2}(\zeta) .
$$

The estimate (4.4) for $f_{\omega}\left(x, x^{\prime} ; t\right)$ is found to hold for $\partial f_{\omega} / \partial \theta\left(x, x^{\prime} ; t\right)$ and this can be used to prove a lemma analogous to Lemma 1 for the sum (4.5). As we would like to work with positive quantities we let $i_{1}$ be the index corresponding to $-l_{1}$, that is $E_{i_{1}}=E_{1}$ and $l_{i_{1}}=-l_{1}$ and similarly for $i_{2}$. Then letting

$$
R_{L, \omega}^{\prime}(s)=\sum_{k \neq 1,2, i_{1}, i_{2}} l_{k}(\omega) e^{-s L^{-2} \eta_{k}(\omega)}
$$

we have

Lemma 2. If $h_{L, \omega}(\zeta)=\sum_{n=1}^{\infty} \zeta^{n} R_{L, \omega}^{\prime}(n \beta)$ then for each $N, \quad h_{L, \omega}(\zeta) \rightarrow$ $\frac{1}{2} \omega a^{2}(2 \pi \beta)^{-3 / 2} g_{3 / 2}(\zeta)$ uniformly for $\zeta \in[0,1], \omega \in[0, N]$ as $L \rightarrow \infty$.

Unlike $\zeta_{1}(L, \omega), \zeta_{2}(L, \omega)$ does not exist for every $\omega$; for example, suppose $\omega<\min \left(\omega_{1}, \omega_{c}\right)$ where $\omega_{c}=2 \bar{\lambda} / \varrho_{c} a^{2}$. Then since $l_{1}(\omega)=0$ we have

$$
L^{-3} \sum_{k=2}^{\infty} l_{k}(\omega) \zeta_{2}(L, \omega)\left(\exp \left\{\beta L^{-2} \eta_{k}(\omega)\right\}-\zeta\right)^{-1}=\bar{\lambda}
$$


For large enough $L$ the sum on the left hand side approaches $\frac{1}{2} \omega a^{2} \varrho_{c}$ and therefore can be made less than $\frac{1}{2} \omega_{c} a^{2} \varrho_{c}^{2}=\bar{\lambda}$. However we can always give an estimate of where $\zeta_{2}(L, \omega)$ must lie for large $L$ if it exists. Define the functions $\alpha$ and $\beta$ on $\mathbb{R}^{+}$by

$$
\begin{aligned}
& \alpha(\omega)= \begin{cases}\theta\left(2 \bar{\lambda} / a^{2} \omega\right), & \omega \geqq \omega_{c}, \\
1, & \omega \leqq \omega_{c} .\end{cases} \\
& \beta(\omega)= \begin{cases}0, & \omega \geqq \omega_{c}, \\
\bar{\lambda}-\frac{1}{2} \omega a^{2} \varrho_{c}, & \omega \leqq \omega_{c} .\end{cases}
\end{aligned}
$$

Then

Theorem 4. For each $\varepsilon>0$ and $N$ positive integer there is an $L_{0}$ such that for all $L>L_{0}$ and $\omega \in\left[0, \omega_{N}\right]$ for which $\zeta_{2}(L, \omega)$ exists and lies in $[0,1]$,

$$
\left|\zeta_{2}(L, \omega)-\alpha(\omega)\right|<\varepsilon
$$

and

$$
\left|b_{L}\left(\omega, \zeta_{2}(L, \omega)\right)-\beta(\omega)\right|<\varepsilon .
$$

Again the proofs of Lemma 2 and Theorem 4 are very similar to the proofs of Lemma 1 and Theorem 1 in [2] and we shall not give them.

Before proceeding any further let us see what to expect for the behaviour of $\zeta(L)$ and $\omega(L)$ for large $L$ and at the same time give a motivation for the proof of Theorem 1. Assume that $\lim _{L \rightarrow \infty} \omega(L)=\omega_{\infty}$ exists and is the value of $\omega$ satisfying

$$
\lim _{L \rightarrow \infty} \zeta_{1}(L, \omega)=\lim _{L \rightarrow \infty} \zeta_{2}(L, \omega),
$$

Then we see from Theorems 3 and 4 that this is equivalent to

$$
\alpha\left(\omega_{\infty}\right)= \begin{cases}\theta(\bar{\varrho}), & \bar{\varrho}<\varrho_{c}, \\ 1, & \bar{\varrho} \geqq \varrho_{c} .\end{cases}
$$

If $\varrho<\varrho_{c}, \theta(\bar{\varrho})<1$ and so $\omega_{\infty}>\omega_{c}$ so that

$$
\alpha\left(\omega_{\infty}\right)=\theta\left(2 \bar{\lambda} / a^{2} \omega_{\infty}\right)
$$

and therefore $\omega_{\infty}=2 \bar{\lambda} / a^{2} \varrho$. If $\varrho \geqq \varrho_{c}$ we obtain no information from (4.6) except that $\omega_{\infty} \leqq \omega_{c}$ so we consider the identity

$$
l_{1}(\omega)=b_{L}(\omega, \zeta) / a_{L}(\omega, \zeta)-\left[l_{2}(\omega)-l_{1}(\omega)\right] c_{L}(\omega, \zeta) / a_{L}(\omega, \zeta) .
$$

Put

$$
\omega=\omega(L), \zeta=\zeta(L)=\zeta_{1}(L, \omega(L))=\zeta_{2}(L, \omega(L)) ;
$$

then

$$
\begin{aligned}
& l_{1}(\omega(L))=b_{L}\left(\omega(L), \zeta_{2}(L, \omega(L))\right) / a_{L}\left(\omega(L), \zeta_{1}(L, \omega(L))\right) \\
& -\left[l_{2}(\omega(L))-l_{1}(\omega(L))\right] c_{L}\left(\omega(L), \zeta_{1}(L, \omega(L))\right) / a_{L}\left(\omega(L), \zeta_{1}(L, \omega(L))\right)
\end{aligned}
$$




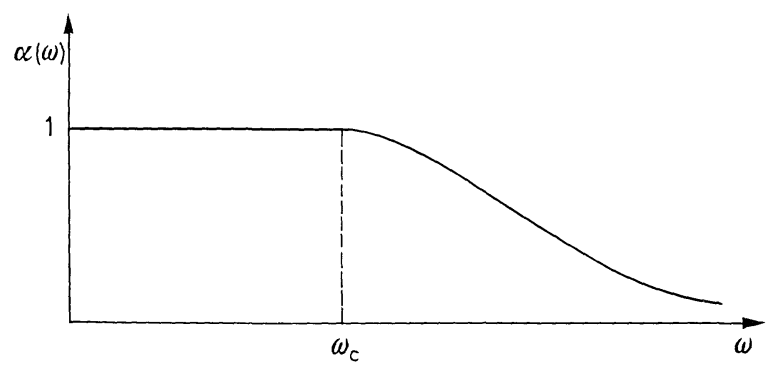

Fig. 2

and therefore we assume that $\omega_{\infty}$ is the value of $\omega$ satisfying

$$
\begin{aligned}
& l_{1}\left(\omega_{\infty}\right)=b_{L}\left(\omega_{\infty}, \zeta_{2}\left(L, \omega_{\infty}\right)\right) / a_{L}\left(\omega_{\infty}, \zeta_{1}\left(L, \omega_{\infty}\right)\right) \\
& -\left[l_{2}\left(\omega_{\infty}\right)-l_{1}\left(\omega_{\infty}\right)\right] c_{L}\left(\omega_{\infty}, \zeta_{1}\left(L, \omega_{\infty}\right)\right) / a_{L}\left(\omega_{\infty}, \zeta_{1}\left(L, \omega_{\infty}\right)\right) .
\end{aligned}
$$

If we make the additional assumption that $\omega_{\infty} \neq \omega_{l}$ for $l=0,1,2, \ldots$, then since $\omega_{\infty} \leqq \omega_{c}$. Theorems 3 and 4 imply that $\omega_{\infty}$ is given by

$$
l_{1}\left(\omega_{\infty}\right)=\left(\bar{\lambda}-\frac{1}{2} \omega_{\infty} a^{2} \varrho_{c}\right) /\left(\bar{\varrho}-\varrho_{c}\right),
$$

or by the intersection of the line

$$
y=\left(\overline{\hat{\lambda}}-\frac{1}{2} \omega a^{2} \varrho_{c}\right) /\left(\bar{\varrho}-\varrho_{c}\right)
$$

with the step function $y=l_{1}(\omega)$. So Theorems 3 and 4 should give us the asymptotic behaviour of $\zeta(L)$ and $\omega(L)$ assuming that the latter are well-behaved. This is ensured by the next theorem whose proof is tedious and is given in [6].

Theorem 5. There is an $N$ and an $L_{0}$ such that for all $L>L_{0}, \omega(L)$ and $\zeta(L)$ exist and $\omega(L)<\omega_{N}$.

Of course this theorem does not ensure the uniqueness of $\omega(L)$ and $\zeta(L)$. But this does not matter since any choice of $\omega(L)$ and $\zeta(L)$ gives the same asymptotic properties. We can now prove Theorem 2.

Proof of Theorem 2. $\varrho \leqq \varrho_{c}$. Since $\omega(L)<\omega_{N}$ for $L>L_{0}$, from Theorem 3 we know that for $L$ sufficiently large,

$$
|\zeta(L)-\theta(\bar{\varrho})|=\left|\zeta_{1}(L, \omega(L))-\theta(\bar{\varrho})\right|<\varepsilon,
$$

and

$$
\left|a_{L}(\omega(L), \zeta(L))\right|=\mid a_{L}\left(\omega(L), \zeta_{1}(L, \omega(L)) \mid<\varepsilon,\right.
$$

that is $\zeta(L) \rightarrow \theta(\varrho), a_{L}(\omega(L), \zeta(L)) \rightarrow 0$. Now suppose that $\bar{\varrho}<\varrho_{c}$ and choose $\varepsilon<1-$ $\theta(\bar{\varrho})$, then as above for large $L$ we have

$$
|\theta(\bar{\varrho})-\alpha(\omega(L))|<\varepsilon,
$$

that is $\alpha(\omega(L))<\theta(\bar{\varrho})+\varepsilon<1$ or $\omega(L)>\omega_{c}$.

Since the function $g_{3 / 2}$ is uniformly continuous on $[0,1]$, for each $\varepsilon>0$ there is a $\delta$ such that $(2 \pi \beta)^{-3 / 2}\left|g_{3 / 2}\left(z_{1}\right)-g_{3 / 2}\left(z_{2}\right)\right|<\varepsilon \bar{Q} / \omega_{N}$ provided $\left|z_{1}-z_{2}\right|<\delta$. By Theorems 3 and 4 , for $L$ sufficiently large

$$
|\zeta(L)-\theta(\bar{\varrho})|=\left|\zeta_{1}(L, \omega(L))-\theta(\bar{\varrho})\right|<\delta / 2
$$


and

$$
\left|\zeta(L)-\theta\left(2 \bar{\lambda} / a^{2} \omega(L)\right)\right|=\left|\zeta_{2}(L, \omega(L))-\theta\left(2 \bar{\lambda} / a^{2} \omega(L)\right)\right|<\delta / 2,
$$

that is

$$
\left|\theta(\bar{\varrho})-\theta\left(2 \bar{\lambda} / a^{2} \omega(L)\right)\right|<\delta .
$$

Therefore

$$
(2 \pi \beta)^{-3 / 2} \mid g_{3 / 2}(\theta(\bar{\varrho}))-g_{3 / 2}\left(\theta\left(2 \bar{\lambda} / a^{2} \omega(L)\right) \mid<\varepsilon \bar{\varrho} \omega_{N}^{-1},\right.
$$

which is the same as

$$
\left|\bar{\varrho}-2 \bar{\lambda} / a^{2} \omega(L)\right|<\varepsilon \bar{\varrho} \omega_{N}^{-1} \text {. }
$$

Therefore

$$
\left|\omega(L)-2 \bar{\lambda} / a^{2} \bar{\varrho}\right|<\omega(L) \bar{\varrho}^{-1} \cdot \varepsilon \bar{\varrho} \omega_{N}^{-1}<\varepsilon,
$$

for sufficiently large $L$. That is $\omega(L) \rightarrow 2 \bar{\lambda} / a^{2} \bar{\varrho}$.

Next consider the case $\bar{\varrho}=\varrho_{c}$. For those values of $L$ which are sufficiently large and for which $\omega(L)>\omega_{c}$ we have as above

$$
\left|\omega(L)-2 \bar{\lambda} / a^{2} \varrho_{c}\right|<\varepsilon .
$$

Suppose now that $\omega(L) \leqq \omega_{c}$, then

$$
\begin{aligned}
& \left|\omega(L)-2 \bar{\lambda} / a^{2} \varrho_{c}\right|=\left(2 / a^{2} \varrho_{c}\right)\left|\bar{\lambda}-\omega(L) a^{2} \varrho_{c} / 2\right| \\
& \leqq \\
& \quad\left(2 / a^{2} \varrho_{c}\right)\left|\bar{\lambda}-\frac{1}{2} \omega(L) a^{2} \varrho_{c}-b_{L}\left(\omega(L), \zeta_{2}(L, \omega(L))\right)\right| \\
& \quad+\left(2 / a^{2} \varrho_{c}\right) \mid b_{L}(\omega(L), \zeta(L) \mid, \\
& \left|b_{L}(\omega(L), \zeta(L))\right| \leqq 2(N+1)\left|a_{L}(\omega(L), \zeta(L))\right|<\frac{1}{4} a^{2} \varrho_{c} \varepsilon
\end{aligned}
$$

for $L$ large, as above, and also for $L$ large by Theorem 4

$$
\mid \bar{\lambda}-\frac{1}{2} \omega(L) a^{2} \varrho_{c}-b_{L}\left(\omega(L), \zeta_{2}(L, \omega(L)) \mid<\frac{1}{4} a^{2} \varrho_{c} \varepsilon .\right.
$$

Therefore

$$
\left|\omega(L)-2 \bar{\lambda} / a^{2} \varrho_{c}\right|<\varepsilon
$$

which completes the proof for $\bar{\varrho} \leqq \varrho_{c}$.

$\bar{\varrho}>\varrho_{c}$. Clearly the proof for $\lim \zeta(L)$ and $\lim a_{L}(\omega(L), \zeta(L))$ holds here as well and we have $\zeta(L) \rightarrow 1$ and $a_{L}(\omega(L), \zeta(L)) \rightarrow \bar{\varrho}-\varrho_{c}$. To obtain $\lim \omega(L)$ we use the identity (4.7). From this identity we see that $\omega(L)$ is a solution of the equation

$$
\begin{aligned}
l_{1}(\omega)= & b_{L}\left(\omega, \zeta_{2}(L, \omega(L))\right) / a_{L}\left(\omega, \zeta_{1}(L, \omega(L))\right) \\
& -\left[l_{2}(\omega)-l_{1}(\omega)\right] c_{L}\left(\omega, \zeta_{1}(L, \omega)\right) / a_{L}\left(\omega, \zeta_{1}(L, \omega)\right) .
\end{aligned}
$$

Put

$$
\begin{aligned}
X_{L}(\omega) & =b_{L}\left(\omega, \zeta_{2}(L, \omega)\right) / a_{L}\left(\omega, \zeta_{1}(L, \omega)\right), \\
Y_{L}(\omega) & =c_{L}\left(\omega, \zeta_{1}(L, \omega)\right) / a_{L}\left(\omega, \zeta_{1}(L, \omega)\right),
\end{aligned}
$$


and

$Z_{L}(\omega)=X_{L}(\omega)+\left[l_{2}(\omega)-l_{1}(\omega)\right] Y_{L}(\omega)$

Then $0<Y_{L}(\omega) \leqq \frac{1}{2}$ and since $\left|l_{2}(\omega)-l_{1}(\omega)\right| \leqq 1$ (Lemma 4$)$,

$\left|Z_{L}(\omega)-X_{L}(\omega)\right|=\left|l_{2}(\omega)-l_{1}(\omega)\right| Y_{L}(\omega) \leqq \frac{1}{2}$.

Now in $\left[\omega_{c}, \omega_{N}\right], a_{L}\left(\omega, \zeta_{1}(L, \omega)\right) \rightarrow \bar{\varrho}-\varrho_{c}$ uniformly by Theorem 3 and for large $L$ independent of $\omega,\left|b_{L}\left(\omega, \zeta_{2}(L, \omega)\right)\right|<\varepsilon$ by Theorem 4 . Therefore $\left|X_{L}(\omega)\right|<\varepsilon$ for large $L$ independent of $\omega$ and consequently $Z_{L}(\omega)<\frac{1}{2}+\varepsilon<1$. On the other hand $l_{1}(\omega) \geqq 1$ for $\omega \geqq \omega_{1}$, so that the equation

$$
l_{1}(\omega)=Z_{L}(\omega)
$$

has no solutions in $\left[\max \left(\omega_{c}, \omega_{1}\right), \omega_{N}\right]$ for large $L$, that is, $\omega(L)<\max \left(\omega_{c}, \omega_{1}\right)$. Also from theorems 3 and 4 we see that if $L$ is sufficiently large, $\omega \in\left[0, \omega_{c}\right]$ and $X_{L}(\omega)$ exists then

$$
\left|X_{L}(\omega)-\left(\bar{\lambda}-\frac{1}{2} \omega a^{2} \varrho_{c}\right) /\left(\bar{\varrho}-\varrho_{c}\right)\right|<\varepsilon .
$$

Figure 3 shows what $Z_{L}(\omega)$ looks like for large $L$.

Let us consider first case (a) of the theorem when the line

$$
y=\left(\bar{\lambda}-\frac{1}{2} \omega a^{2} \varrho_{c}\right) /\left(\bar{\varrho}-\varrho_{c}\right)
$$

intersects the step function $y=l_{1}(\omega)$ at $\omega^{\prime} \in\left(\omega_{l}, \omega_{l+1}\right)$ for some $l \geqq 0$. We shall assume at first that $\omega_{c} \geqq \omega_{1}$ so that $l \geqq 1, \omega_{l}<\omega^{\prime}<\omega_{c}$ and for large $L, \omega(L)<\omega_{c}$. For $\omega \in\left[0, \omega_{c}\right)$ and large $L$ by (4.8) and (4.9) we have

$$
\begin{aligned}
Z_{L}(\omega) & >\left(\bar{\lambda}-\frac{1}{2} \omega a^{2} \varrho_{c}\right) /\left(\bar{\varrho}-\varrho_{c}\right)-\frac{1}{2}-\varepsilon \\
& >\left(\bar{\lambda}-\frac{1}{2} \omega^{\prime} a^{2} \varrho_{c}\right) /\left(\bar{\varrho}-\varrho_{c}\right)-\frac{1}{2}-\varepsilon=l-\frac{1}{2}-\varepsilon .
\end{aligned}
$$

But for $\omega \in\left[0, \omega_{l}\right), l_{1}(\omega) \leqq l-1$ which means that for large $L, \omega(L) \geqq \omega_{l}$. Similarly for sufficiently large $L, \omega(L)<\omega_{l+1}$. Now choose $\delta>0$ such that the equations

$$
l_{1}(\omega)=\left(\bar{\lambda}-\frac{1}{2} \omega a^{2} \varrho_{c}\right) /\left(\bar{\varrho}-\varrho_{c}\right) \pm 2 \delta
$$

have solutions $\omega \pm$ satisfying

$$
\omega_{l}<\omega_{-}<\omega^{\prime}<\omega_{+}<\omega_{l+1}
$$

and put $\bar{a}=\omega_{-} \wedge a_{l}$ and $\bar{b}=\omega_{+} \vee b_{l}$ where $a_{l}$ and $b_{l}$ are as in Lemma (4). For $\omega \in\left[\omega_{l}, \bar{a}\right), l_{2}(\omega)-l_{1}(\omega)=1$ and therefore for large $L$

$$
\begin{aligned}
Z_{L}(\omega) & \geqq X_{L}(\omega) \geqq\left(\bar{\lambda}-\frac{1}{2} \omega a^{2} \varrho_{c}\right) /\left(\bar{\varrho}-\varrho_{c}\right)-\delta \\
& \geqq\left(\bar{\lambda}-\frac{1}{2} \omega a_{-} \varrho^{2} \varrho_{c}\right) /\left(\bar{\varrho}-\varrho_{c}\right)-\delta=l+\delta
\end{aligned}
$$

which means that $\omega(L) \geqq \bar{a}$ for large $L$ since $l_{1}(\omega)=l$ for $\omega \in\left[\omega_{l}, \bar{a}\right)$. Similarly $\omega(L) \leqq \bar{b}$ and therefore $\omega(L) \in[\bar{a}, \bar{b}]$. Now by Theorem $3, c_{L}\left(\omega, \zeta_{1}(L, \omega)\right) \rightarrow 0$ uniformly on $[\bar{a}, \bar{b}]$, that is

$$
\left|Z_{L}(\omega(L))-\left(\bar{\lambda}-\frac{1}{2} \omega(L) a^{2} \varrho_{c}\right) /\left(\bar{\varrho}-\varrho_{c}\right)\right|<\varepsilon
$$

for large $L$. But $l_{1}(\omega)=l$ if $\omega \in[\bar{a}, \bar{b}]$ so that

$$
Z_{L}(\omega(L))=l_{1}(\omega(L))=l=\left(\bar{\lambda}-\frac{1}{2} \omega^{\prime} a^{2} \varrho_{c}\right) /\left(\bar{\varrho}-\varrho_{c}\right) .
$$




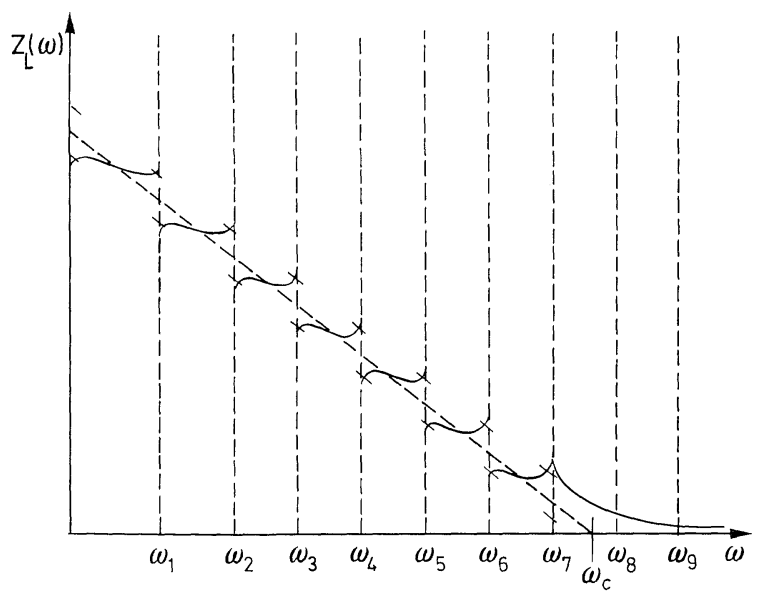

Fig. 3

Therefore $\left|\omega(L)-\omega^{\prime}\right|<2 \varepsilon\left(\bar{\varrho}-\varrho_{c}\right) / a^{2} \varrho_{c}$ for all $L$ sufficiently large, which means that $\omega(L) \rightarrow \omega^{\prime}$. Clearly $l_{1}(\omega(L)) \rightarrow l$ and $c_{L}(\omega(L), \zeta(L)) \rightarrow 0$.

If $\omega_{c}<\omega_{1}$ we have $\omega^{\prime}=\omega_{c}$. Then exactly as above, we can prove that $\omega(L)>$ $\omega_{c}-\varepsilon$ for large $L$ and as in the case for $\bar{\varrho} \leqq \varrho_{c},|1-\alpha(\omega(L))|<\varepsilon^{\prime}$ for large $L$, that is, $\omega(L)<\omega_{c}+\varepsilon$. Thus we have $\omega(L) \rightarrow \omega^{\prime}=\omega_{c}, l_{1}(\omega(L)) \rightarrow 0$ and $c_{L}(\omega(L), \zeta(L)) \rightarrow 0$.

We now consider case (b) when the line

$$
y=\left(\bar{\lambda}-\frac{1}{2} \omega a^{2} \varrho_{c}\right) /\left(\bar{\varrho}-\varrho_{c}\right)
$$

passes through the gap at $\omega=\omega_{l}$ a distance $t$ from the bottom of the gap. Here we necessarily have $\omega_{c} \geqq \omega_{1}$ and $\omega_{l}<\omega_{c}$. We shall take $\frac{1}{2}<t \leqq 1$. The case when $0 \leqq t<\frac{1}{2}$ is similar. For $L$ large and $\omega<\omega_{l}$

$$
Z_{L}(\omega)>\left(\bar{\lambda}-\frac{1}{2} \omega_{l} a^{2} \varrho_{c}\right) /\left(\bar{\varrho}-\varrho_{c}\right)-\frac{1}{2}-\left(t-\frac{1}{2}\right)=l-1 .
$$

But $l_{1}(\omega) \leqq l-1$ for $\omega<\omega_{l}$ which means that $\omega(L) \geqq \omega_{i}$. Similarly $\omega(L)<\omega_{l+1}$ for large $L$ and we can prove as in case (a) that for large $L, \omega(L) \leqq b_{l}$. Now suppose $\varepsilon>0$ and put

$$
\sigma=l-\left(\bar{\lambda}-\frac{1}{2}\left(\omega_{l}+\varepsilon\right) a^{2} \varrho_{c}\right) /\left(\bar{\varrho}-\varrho_{c}\right)
$$

then if $\omega \in\left[\omega_{l}+\varepsilon, b_{l}\right]$

$$
\left(\bar{\lambda}-\frac{1}{2} \omega a^{2} \varrho_{c}\right) /\left(\bar{\varrho}-\varrho_{c}\right) \leqq\left(\bar{\lambda}-\frac{1}{2}\left(\omega_{l}+\varepsilon\right) a^{2} \varrho_{c}\right) /\left(\bar{\varrho}-\varrho_{c}\right)=l-\sigma .
$$

But in $\left[\omega_{l}+\varepsilon, b_{l}\right], c_{L}\left(\omega, \zeta_{1}(L, \omega)\right) \rightarrow 0$ uniformly by Theorem 3 which means that for $L$ sufficiently large

$$
Z_{L}(\omega)<\left(\bar{\lambda}-\frac{1}{2} \omega a^{2} \varrho_{c}\right) /\left(\bar{\varrho}-\varrho_{c}\right)+\sigma<l
$$

for all $\omega \in\left[\omega_{l}+\varepsilon, b_{l}\right]$, which leaves us with $\omega_{l} \leqq \omega(L)<\omega_{l}+\varepsilon$ for large $L$. Therefore $\omega(L) \rightarrow \omega_{l}, l_{1}(\omega(L)) \rightarrow l, l_{2}(\omega(L)) \rightarrow l-1$ and since for large $L, l_{1}(\omega(L))=l, l_{2}(\omega(L))=$ 
l-1 we have

$$
\begin{aligned}
c_{L}(\omega(L), \zeta(L))= & l a_{L}(\omega(L), \zeta(L))-b_{L}(\omega(L), \zeta(L)) \\
& \rightarrow l\left(\bar{\varrho}-\varrho_{c}\right)+\frac{1}{2} \omega_{l} a^{2} \varrho_{c}-\bar{\lambda} \\
= & (1-t)\left(\bar{\varrho}-\varrho_{c}\right) .
\end{aligned}
$$

Finally we turn to the case $t=\frac{1}{2}$. As above we can prove that for large $L$, $\omega_{l}-\varepsilon<\omega(L)<\omega_{l}+\varepsilon$ so that $\omega(L) \rightarrow \omega_{l}$. Now suppose that $\omega_{l} \leqq \omega(L)<\omega_{l}+\varepsilon$ then $l_{1}(\omega(L))=l, l_{2}(\omega(L))=l-1$ and

$$
c_{L}(\omega(L), \zeta(L))=l a_{L}(\omega(L), \zeta(L))-b_{L}(\omega(L), \zeta(L)) .
$$

Therefore since $\bar{\lambda}=\left(l-\frac{1}{2}\right)\left(\bar{\varrho}-\varrho_{c}\right)+\frac{1}{2} \omega_{l} a^{2} \varrho_{c}$

$$
\left|\mathcal{C}_{L}(\omega(L), \zeta(L))-\frac{1}{2}\left(\bar{\varrho}-\varrho_{c}\right)\right|<\varepsilon^{\prime}
$$

for large $L$. Similarly if $\omega_{l}-\varepsilon<\omega(L)<\omega_{l}$ so that $c_{L}(\omega(L), \zeta(L)) \rightarrow \frac{1}{2}\left(\bar{\varrho}-\varrho_{c}\right)$ as $L \rightarrow \infty$, which completes the proof.

To prove Theorem 1 we define

$$
T_{L, \omega}^{\prime}(s)=\sum_{k=3}^{\infty} e^{-s L^{-2} \eta_{k}(\omega)}\left|\left\langle\phi_{k, \omega}^{L}, h\right\rangle\right|^{2}
$$

and

$$
T_{L, \omega}(s)=\sum_{k=1}^{\infty} e^{-s L^{-2} \eta_{k}(\omega)}\left|\left\langle\phi_{k, \omega}^{L}, h\right\rangle\right|^{2}
$$

for $h$ in the Schwartz space $\mathscr{D}$ and $L>L_{0}$ where $L_{0}$ is chosen so that the support of $h$ is contained in $\Lambda^{L_{0}}$. Putting

$$
K(s)=\int_{\mathbb{R}^{3} \times \mathbb{R}^{3}} \bar{h}(x) h(y) \exp \left\{-\|x-y\|_{1}^{2} / 2 s\right\} d x d y
$$

we can use the estimate (4.4) to prove the following Lemma. The proof is very similar to that of Lemma 2 in [2].

Lemma 3. Suppose that $\lim _{L \rightarrow \infty} \zeta(L)=\zeta_{0} \in[0,1]$ and $\lim _{L \rightarrow \infty} \omega(L)$ exists. Then

$$
\lim _{L \rightarrow \infty} \sum_{n=1}^{\infty} \zeta(L)^{n} T_{L, \omega(L)}^{\prime}(n \beta)=\sum_{n=1}^{\infty} \zeta_{0}^{n} K(n \beta) .
$$

The details of the proof of Theorem 1 are similar to those of Theorem 1 of 2 and so we omit them. The proof of Theorem 5 is based on the following lemma whose proof is straightforward.

Lemma 4. For each $l \geqq 1$ there are numbers $a_{l}$ and $b_{l}$ satisfying:

(1) $\omega_{l}<a_{l} \leqq b_{l}<\omega_{l+1}$

(2) $l_{2}(\omega)=l-1$ for $\omega_{l} \leqq \omega<a_{l}$

$$
=l \quad \text { for } \quad b_{l} \leqq \omega<\omega_{l+1} \text {. }
$$

(3) $a_{l}=b_{l}$ implies that $a_{l}=b_{l}=\frac{1}{2}\left(\omega_{l}+\omega_{l+1}\right)$ while $a_{l}<b_{l}$ implies that $a_{l}<\frac{1}{2}\left(\omega_{l}+\right.$ $\left.\omega_{l+1}\right)$.

(4) If $a_{l} \leqq \omega<b_{l}$ then $l_{2}(\omega)=l$.

There is also an $a_{0}$ satisfying $0 \leqq a_{0}<\omega_{1}$ and

$$
\begin{aligned}
& l_{2}(\omega)=0 \text { for } 0 \leqq \omega<a_{0} \text {, } \\
& =1 \text { for } a_{0} \leqq \omega<\omega_{1} \text {. }
\end{aligned}
$$




\section{References}

1. Kac, M.: Private communication, 1971

2. Lewis, J.T., Pulè, J.V.: Commun. math. Phys. 36, 1 (1974)

3. Blatt, J.M., Butler, S.T.: Phys. Rev. 100, 476 (1955)

4. Putterman, S. J., Kac, M., Uhlenbeck, G.E.: Phys. Rev. Lett. 29, 546 (1972)

5. Andronikashivili, E.L., Mamaladze, Yu. G.: Rev. Mod. Phys. 38, 567 (1966)

6. Pulè,J.V.: D. Phil. Thesis, Oxford: 1972

7. Watson, G. N.: A Treatise on the Theory of Bessel Functions. Cambridge: University Press 1944

8. Feynman, R.P.: Progress in Low Temperature Physics, Vol. 1, Chap. 2, Amsterdam: North Holland (1955)

9. Blatt, J.M., Butler,S.T., Schafroth, M.R.: Phys. Rev. 100, 481 (1955)

Communicated by G. Gallavotti

Received October 22, 1974; in revised form July 2, 1975 
